Revista de Psicología y Educación / Journal of Psychology and Education, 2021, 16(2), 173-182 (www.rpye.es) Doi: https://doi.org/10.23923/rpye2021.02.209

ISSN: $1699-9517$

\title{
Estatus social, búsqueda de popularidad y narcisismo en la conducta agresiva del acoso escolar y el ciberacoso
}

\author{
Eva M. Romera*, José A. Casas, Yolanda Muñoz y Rosario Ortega-Ruiz \\ Universidad de Córdoba
}

\begin{abstract}
Resumen: Las investigaciones sobre acoso y ciberacoso han señalado la tendencia de algunos adolescentes a realizar comportamientos agresivos para conseguir un estatus de cierta relevancia dentro del grupo de iguales. Los objetivos del trabajo que se presenta han buscado, una vez descritos los niveles de popularidad y narcisismo, explorar la posible relación entre las metas de popularidad y el narcisismo y la implicación de los adolescentes en agresión en bullying y cyberbullying. Participaron 1728 estudiantes (48,1\% chicas) de Educación Secundaria Obligatoria de Andalucía (España). Se utilizaron los siguientes instrumentos de autoinforme: el European Bullying Intervention Project Questionnaire (EBIPQ), European Cyberbullying Intervention Project Questionnaire; la Hypersensitive Narcissism Scale y la Social Achievement Goal Scale. Análisis descriptivos y de regresión lineal señalaron que los chicos puntuaron más alto en ambos tipos de agresión y que las chicas se mostraron significativamente más narcisistas. El narcisismo y la popularidad percibida influyeron en la implicación tanto en acoso escolar como en ciberacoso. Se discuten los resultados tanto en base a la importancia de la imagen dentro del grupo de los iguales para comprender la implicación en agresión y ciberagresión, como a la relevancia educativa de los mismos.
\end{abstract}

Palabras clave: Agresión, Ciberagresión, Motivación social, Popularidad, Narcisismo, Adolescencia.

\section{Social status, popularity goals, and narcissism in bullying and cyberbullying perpetration}

Abstract: Research on bullying and cyberbullying has pointed out the tendency of some adolescents to perform aggressive behaviors to achieve a relevant status within the peer group. The objectives of this study were to describe popularity and narcissism levels in aggression and cyber-agression, and their relationship with bullying and cyberbullying perpetration. A total of 1728 students from Andalusia (48.1\% girls) attending secondary school participated in the study. The self-report instruments were the European Bullying Intervention Project Questionnaire, the European Cyberbullying Intervention Project Questionnaire, the Hypersensitive Narcissism Scale, and the Social Achievement Goal Scale. Descriptive and linear regression analyses indicated that boys scored higher in both types of aggression, and girls were significantly more narcissistic. Narcissism and perceived popularity influenced both bullying and cyberbullying involvement. The results are discussed both concerning the importance of image within the peer group to understand engagement in aggression and cyber-aggression, as well as their educational relevance.

Key words: Agression, Cyberagression, Social motivation, Popularity, Narcissism, Adolescence.

El acoso escolar es un fenómeno cuya dinámica social implica un juego de roles en el que participan al menos la víctima, el agresor - los agresores y uno o varios espectadores

Recibido: 08/04/2021 - Aceptado: 04/06/2021 - Avance online: 16/06/2021

*Correspondencia: Eva M. Romera

Universidad de Córdoba, España

Dirección: 14071, Córdoba, España.

E-mail: eva.romera@uco.es

Romera, E. M. Casas, J. A., Muñoz, Y y Ortega-Ruiz, R. (2021). Estatus social, búsqueda de Romera, E. M., Casas, J. A., Muñoz, Y. y Ortega-Ruiz, R. (2021). Estatus social, búsqueda de de Psicología y Educación, 16(2), 173-182, htpps://doi.org/10.23923/rpye2021.02.209
(Hymel et al., 2015). Olweus definió el bullying como una situación en la que "un alumno está siendo maltratado o victimizado cuando él o ella está expuesto repetidamente y a lo largo del tiempo a acciones negativas de otro o un grupo de estudiantes" (Olweus, 1999; p. 10). Estas acciones pueden manifestarse de forma verbal, física o relacional.

1699-9517/@ 2021 Asociación Científica de Psicología y Educación (ACIPE). Publicado por Consejo General de Colegios Oficiales de Psicólogos, España. Este es un artículo Open Access bajo la CC BY-NC-ND licencia (http://creativecommons.org/licencias/bync-nd/4.0/). 
En la actualidad, el uso de los dispositivos digitales ha supuesto una nueva forma de relacionarnos (Fernández-Montalvo et al., 2015). A su vez, la rápida evolución de las Tecnologías de la Información y Comunicación (TIC) y el fácil acceso de niños y adolescentes a estas tecnologías han dado lugar a que, junto a las nuevas formas de comunicarnos y socializarnos, se incluyan conductas no exentas de riesgo asociadas al uso indebido de los dispositivos digitales, entre ellos el ciberacoso (Smahel et al., 2020).

El ciberacoso ha sido reconocido por un amplio número de investigaciones como una forma indirecta de acoso escolar, a la que se añaden las características específicas de la ciberconduta (Ortega-Ruiz \& Zych, 2016). Smith et al. (2008) subrayan que el ciberacoso es un acto agresivo e intencionado llevado a cabo de manera repetida y constante a lo largo del tiempo, mediante el uso de formas de contacto electrónicas, por parte de un grupo o de un individuo contra una víctima que no puede defenderse fácilmente. Tanto en el acoso como en el ciberacoso se reconoce la intencionalidad de hacer daño y la reproducción de un esquema de dominio y sumisión de carácter inmoral que causa daño a la víctima y envilece al agresor (Romera et al., 2019).

Los estudios sobre acoso y ciberacoso han identificado dos dimensiones para la comprensión de ambos fenómenos, victimización y agresión -on/off line- (OrtegaRuiz et al., 2016). Las investigaciones han reconocido un solapamiento entre las personas que participan en el acoso tradicional y el cibernético, en ambas dimensiones (Garmendia et al., 2019), así como consecuencias negativas asociadas a ambos fenómenos (Garaigordobil, 2011; Zych et al., 2015). Un amplio número de estudios ha prestado atención a los factores de riesgo y protección que se asocian con el comportamiento agresivo y de victimización entre los escolares. Entre dichos factores, tienen un papel principal las propias relaciones que se gestan en el grupo de los iguales, la dinámica relacional y, más concretamente, el estatus social que los escolares implicados adquieren en el marco de esas relaciones. En este sentido, la popularidad se ha identificado como un factor determinante (Pouwels et al., 2018). La popularidad es el reconocimiento por parte de los demás de la relevancia de cada individuo. Pero dicho reconocimiento del individuo no siempre coincide con la dimensión que los otros valoran, de ahí la importancia de tener en cuenta la popularidad percibida. La popularidad percibida indica la valoración de uno mismo sobre el impacto en el dominio social y la influencia social que se ejerce sobre los demás en un grupo, en el aula por ejemplo (Laninga-Wijnen et al., 2019). Ser o no ser popular parece que se convierte en algo importante para algunos escolares. Existen diferentes factores que contribuyen a la condición de ser populares o no, algunos de los cuales han recibido atención investigadora desde hace bastante tiempo. Factores como el aspecto físico, la sociabilidad y el carácter abierto y en general la inteligencia social han sido destacados como rasgos con tendencia a gozar de popularidad (Kleiser \& Mayeux, 2021).

Atendiendo a la implicación en los fenómenos de acoso y ciberacoso, algunas investigaciones indican que las víctimas y los agresores victimizados reportan menor nivel de popularidad entre sus iguales (Cerezo et al., 2015; Holt \& Espelage, 2012). Respecto de los agresores, investigaciones previas indican que la agresión puede ser interpretada como una forma de conseguir el éxito social dentro del grupo (Dumas et al., 2019). En esta línea, diferentes estudios han señalado que los chicos y chicas que están movidos por metas de popularidad, en las que se busca el reconocimiento social, son más propensos a desarrollar conductas agresivas (Malamut et al., 2020). En esta línea, diferencias de sexo han sido puestas en evidencia: si bien se ha reconocido que la popularidad está más relacionada con la agresión relacional de las chicas (Cillessen \& Mayeux, 2004), investigaciones recientes señalan que los efectos de la búsqueda de popularidad relacionada con el comportamiento agresivo son más notorios en los chicos (Cillessen et al., 2014). 
No cabe duda de que la tendencia a buscar popularidad puede depender de las características de personalidad de cada individuo. En este sentido, ser más o menos narcisista, en el sentido subclínico, es decir, disponer de rasgos narcisísticos de personalidad podría estar muy relacionado con el deseo de ser reconocido socialmente, de ser relevante en el interior del grupo de iguales (Poorthuis et al., 2021). El narcisismo ha sido identificado como una variable relacionada con la popularidad. La motivación de los agresores de acoso y ciberacoso para ganar poder, dominio y prestigio sobre los demás puede ser debido precisamente a altos niveles de narcisismo (Miller et al., 2007; Morf \& Rhodewalt, 2001). La creencia de que uno es único y más importante que los demás, y una necesidad excesiva de aprobación y admiración parece alimentar dicha motivación (Miller et al., 2007). Rasgos de personalidad asociados, como la arrogancia, han sido descritos en aquellos que abusan o victimizan a sus iguales (Morf \& Rhodewalt, 2001; Thomaes \& Brummelman, 2015).

El Manual Diagnóstico y Estadístico de los Trastornos Mentales - 5 (DSM-5; American Psychiatric Association, 2013) incluye la necesidad de atención y admiración, así como la hipersensiblidad a la evaluación de los demás, como rasgos básicos de la personalidad narcisística. La investigación señala que el constructo narcisismo es controvertido y en todo caso es multidimensional, que incluye, por un lado, competencia para la adaptación social incluyendo el liderazgo y actitudes de superioridad, y, por otro, incompetencia, mala adaptación social, incluyendo exhibicionismo o arrogancia y búsqueda del dominio sobre otros (Krizan \& Herlache, 2018). Golmaryami y Barry (2010) encontraron asociaciones entre el desajuste social del narcisismo y la agresión relacional. En un reciente metaanálisis se confirma que el narcisismo es un claro precursor de la agresión en todas sus formas (Kiærvik \& Bushman, 2021).

A pesar de que los estudios sobre narcisismo tienden a señalar diferencias de sexo, no todas las investigaciones apuntan hacia esta tendencia (Pauletti et al., 2012). Los chicos son más propensos a ser narcisistas (Reijntjes et al., 2016). Pero también se ha argumentado que los narcisistas suelen hacer un mayor uso de la agresión indirecta, una forma de violencia que se ha descrito como más característica de las chicas (Card et al., 2008; Golmaryamia \& Barrry, 2010). Por tanto, se requiere continuar indagando en las posibles diferencias de sexo respecto de la conducta o rasgos narcisísticos de los adolescentes.

A pesar de la relevancia reconocida en la comprensión de la conducta agresiva en el acoso y ciberacoso de la popularidad percibida, la motivación de popularidad y el narcisismo, no se ha explorado cómo estas variables influyen conjuntamente en ambos fenómenos, ni cuáles son más relevantes en relación a la agresión off/online. Por ello, se plantean los siguientes objetivos de este estudio: 1. Describir en una muestra de adolescentes andaluces los niveles de agresión y ciberagresión, popularidad y narcisismo, analizando las homogeneidades o diferencias sexuales. 2. Explorar la relación entre popularidad, metas de popularidad y narcisismo con la agresión y ciberagresión, analizando a su vez las similitudes y diferencias entre ambos fenómenos.

Sustentamos los objetivos en las siguientes hipótesis: a) serán los chicos quienes muestren mayores niveles de implicación en agresión, narcisismo y popularidad en comparación con las chicas; b) percibirse como populary mostrar rasgos narcisistas influirá positivamente en la implicación en agresión y ciberagresión; y c) la agresión mostrará una asociación positiva con la ciberagresión y viceversa.

\section{MÉTODO}

\section{PARTICIPANTES}

En este estudio han participado 1728 escolares (48\% chicas) de la comunidad Autónoma de Andalucía (España), de Educación Secundaria Obligatoria (E.S.O.), distribuidos entre: $1^{\circ}$ de $\operatorname{ESO}(22,6 \%), 2^{\circ}$ de ESO $(26,5 \%), 3^{\circ}$ de ESO $(24,6 \%)$ y $4^{\circ}$ de ESO $(26,2 \%)$. Las edades estuvieron comprendidas entre los 11 y los 18 años, con una media 
de $13,63(D T=1,32)$. Participaron un total de 20 centros escolares de áreas rurales y urbanas (62\% públicos y $38 \%$ privado-concertados). El muestreo fue probabilístico aleatorio en función de los centros escolares que han participado.

\section{INSTRUMENTOS}

La subescala de agresión de la European Bullying Intervention Project Questionnaire (EBIPQ) (Ortega-Ruiz et al., 2016). Este es un instrumento de autoinforme compuesto inicialmente por 14 ítems tipo Likert de cinco opciones: $0=$ no; $1=$ sí, una o dos veces; $2=$ sí, una o dos veces al mes; 3 = sí, alrededor de una vez a la semana; y $4=$ sí, más de una vez a la semana. En la presente investigación solo se ha usado el factor de agresión, compuesto por 7 ítems (ej.: He amenazado a alguien). Una mayor puntuación indicó un mayor nivel de implicación en agresión. La consistencia interna de la escala de agresión $(\alpha=.91)$ fue adecuada en este estudio.

La subescala de agresión de la European Cyberbullying Intervention Project Questionnaire (ECBIPQ) (Del Rey et al., 2015; validado en español en Ortega-Ruiz et al., 2016). Este instrumento se compone de dos dimensiones: cibervictimización y ciberagresión. Esta última es la que se ha usado en la presente investigación. Está compuesta por 13 ítems, como por ejemplo He creado una cuenta falsa para hacerme pasar por otra persona o He excluido o ignorado a alguien en una red social o chat. Con cinco opciones de respuesta: $0=$ no; $1=$ sí, una o dos veces; 2 = sí, una o dos veces al mes; 3 = sí, alrededor de una vez a la semana; y 4 = sí, más de una vez a la semana. Una mayor puntuación indica una mayor implicación en ciberagresión. La consistencia interna de la escala en esta investigación fue de $\alpha=.93$.

Escala de Hipersensibilidad Narcisista (The Hypersensitive Narcissism Scale, HSNS) (Hending \& Cheek, 1997; validada en español en Ripoll et al., 2010). Este instrumento consta de 10 ítems que se responden en una escala tipo Likert de 5 puntos $(1=$ totalmente falso, 2 = falso, 3 = neutro, 4 = cierto, 5 = totalmente cierto). El HSNS es una prueba psicométrica que valora el narcisismo hipersensible o encubierto (ej: Tengo bastante con mis cosas como para preocuparme por los de otra gente; Puedo llegar a quedarme completamente distraído o aislado pensando en mis asuntos, mi salud, mis preocupaciones o mis relaciones con los demás). Una mayor puntuación indicaba un mayor nivel de narcisismo. La escala mostró buena consistencia interna con la muestra de estudio $(\alpha=.73)$.

Escala de Metas Sociales (Social Achievement Goal Scale) (Ryan \& Shim, 2008; validada en español por Herrera-López et al., 2016). Este instrumento consta de 12 ítems estructurados en tres factores: metas de desarrollo social, metas de popularidad y metas de evitación social. Para este estudio se utilizó la escala de metas de popularidad (ej.: Es importante para mí que otros piensen en mí como popular). Las respuestas se midieron utilizando una escala tipo Likert con 7 opciones de respuesta sobre el grado de acuerdo: 1 (no es cierto) a 7 (muy cierto). Una mayor puntuación en la escala indicaba un mayor nivel de metas de popularidad. La consistencia interna de la escala con la muestra de estudio fue óptima ( $\alpha=.83)$.

Popularidad percibida. Se utilizaron dos ítems para su medición: Soy popular entre los chicos y Soy popular entre las chicas. Las opciones de respuesta a estos ítems indicaron el grado de acuerdo y oscilaron entre 1 (falso) y 6 (verdadero). Una mayor puntuación indicaba un mayor nivel de popularidad percibida.

Las puntuaciones totales en cada una de las dimensiones de estudio se calcularon en base a la media de los ítems que las componen.

\section{PROCEDIMIENTO}

El diseño metodológico fue transversal mediante un estudio ex post facto retrospectivo (Montero \& León, 2007). Los datosson el resultado de la cumplimentación de los instrumentos en centros escolares de Andalucía, a los cuales se había solicitado permiso mediante un carta o correo a la dirección del centro al cual se le remitió información exhaustiva del estudio a 
realizar, bajo las normas de anonimato y reserva de la intimidad del alumnado participante y bajo el permiso de la familia o tutores legales (tal como recoge la normativa de la Administración Educativa de Andalucía y los principios generales de la Declaración de Helsinki). El estudio fue aprobado por el Comité de Bioética y Bioseguridad de la Universidad de Córdoba. La administración de los cuestionarios estuvo a cargo de miembros del equipo de investigación que, en sesión presencial, explicaron a los alumnos y alumnas cómo contestar. Se insistió en la importancia de mostrar sinceridad en sus respuestas. El tiempo de cumplimentación osciló entre los 45 y 50 minutos.

\section{ANÁLISIS DE DATOS}

Para dar respuesta al primer objetivo se realizaron los análisis descriptivos simples de todas las variables estudiadas. La prueba de diferencia de medias $t$ de Student se utilizó para observar las diferencias significativas entre los chicos y chicas. Para el tamaño del efecto en las comparaciones dos a dos se informó del estadístico d de Cohen. Un valor por debajo de .50 se consideró un efecto bajo, entre .50 y .80 un efecto moderado y por encima de .80 un efecto grande. Para la consecución del segundo objetivo se llevaron a cabo dos modelos de regresión lineal por el método de pasos sucesivos, para encontrar la relación entre las variables independientes y la agresión en acoso escolar y en ciberacoso. Se utilizó el programa estadístico SPSS (versión 23).

\section{RESULTADOS}

En relación con el primer objetivo, a la búsqueda de validar la primera hipótesis, se ha realizado el análisis t de Student para observar las diferencias entre chicos y chicas respecto de su implicación en agresión, narcisismo y popularidad, existiendo diferencias en todas las variables. Es decir, los chicos mostraron una implicación más alta significativamente en acoso escolar y ciberacoso, y una mayor puntuación en la popularidad, mientras las chicas presentaron significativamente mayor puntuación en narcisismo (ver Tabla 1).

\begin{tabular}{|c|c|c|c|c|c|c|c|c|}
\hline \multicolumn{9}{|c|}{$\begin{array}{c}\text { Tabla } 1 \\
\text { Diferencia de medias entre chicos y chicas }\end{array}$} \\
\hline & $M$ & $D T$ & Sexo & $M$ & $D T$ & $t$ & $p$ & $d$ \\
\hline \multirow{3}{*}{$\begin{array}{l}\text { Agresión } \\
\text { bullying }\end{array}$} & & & Chico & 0.40 & 0.59 & \multirow[b]{2}{*}{7.86} & \multirow[b]{2}{*}{$<.001$} & \multirow[b]{2}{*}{036} \\
\hline & 0.31 & 0.47 & & & & & & \\
\hline & & & Chica & 0.23 & 0.32 & & & \multirow[b]{3}{*}{0.19} \\
\hline \multirow{3}{*}{$\begin{array}{l}\text { Agresión } \\
\text { cyberbullying }\end{array}$} & & & Chico & 0.17 & 0.40 & \multirow[b]{2}{*}{4.54} & & \\
\hline & 0.13 & 0.30 & & & & & $<.001$ & \\
\hline & & & Chica & 0.11 & 0.19 & & & \multirow[b]{3}{*}{0.09} \\
\hline \multirow{3}{*}{ Narcisismo } & & & Chico & 2.35 & 0.71 & \multirow[b]{2}{*}{-2.01} & & \\
\hline & 2.38 & 0.70 & & & & & .045 & \\
\hline & & & Chica & 2.41 & 0.69 & & & \\
\hline \multirow{3}{*}{$\begin{array}{l}\text { Metas } \\
\text { Popularidad }\end{array}$} & & & Chico & 2.51 & 1.01 & \multirow[b]{2}{*}{7.73} & & \multirow[b]{2}{*}{0.36} \\
\hline & 2.33 & 1.00 & & & & & $<.001$ & \\
\hline & & & Chica & 2.16 & 0.96 & & & \\
\hline
\end{tabular}


En un segundo paso, se utilizó el procedimiento de regresión lineal múltiple, tomando como variable criterio o dependiente la agresión en bullying y como variables predictoras la agresión en cyberbullying, las metas de popularidad, el narcisismo y la popularidad percibida. El modelo conseguido $\left(F_{4,1728}=142.86, p=.001\right)$, mostró una $R^{2}$ de .24 , siendo las variables predictoras: agresión en cyberbullying $<<A$.CB $>>(t=19.67, p<$ $.001)$, metas de popularidad $<<M P>>(t=$ 3.82, $p<.001)$, narcisismo $<<N>>(t=$ 5.38, $p<.001$ ), y popularidad percibida entre los chicos $<<$ PChicos $>>(t=4.271, p<$ .001 ). El valor de Durbin-Watson fue de 1,91 y los valores de inflación de la varianza (FIV) oscilaron entre 1.05 y 1.20 en las variables independientes.

Agresión Bullying $=-.16+.64 \mathrm{ACB}+.04$ MP + .07 N + .03 PChicos

El segundo modelo de regresión lineal tuvo como variable dependiente la agresión en cyberbullying. Las variables independientes fueron agresión en bullying, narcisismo y popularidad entre los chicos. El mejor modelo $(F=165.56 ; p<.001)$ obtuvo una $R^{2}$ de .22 , siendo las variables predictoras agresiónen bullying $<<A B>>(t=19.80, p<.001)$, narcisismo $<<N>>(t=3.74, p=.000)$, y popularidad percibida entre los chicos $<<$ PChicos $>>(t=3.12, p<.001)$. El valor de Durbin-Watson fue de 1,90 y los valores de FIV oscilaron entre 1.04 y 1.07 para las variables independientes.

Agresión Cyberbullying $=-.08+.28 \mathrm{AB}+$ $.03 N+.01$ PChicos

\section{DISCUSIÓN}

La finalidad de esta investigación consistió en analizar los niveles de implicación en agresión y ciberagresión, así como la relación que hay entre ambos a través del análisis descriptivo y de regresión lineal de las variables de estudio.

La primera hipótesis se vio confirmada parcialmente. Si bien los chicos mostraron mayores niveles de agresión, ciberagresión y popularidad, las chicas mostraron puntuaciones más altas en narcisismo. La mayor implicación de los chicos en agresión en acoso escolar y ciberacoso coincide con la mayoría de estudios sobre ambos fenómenos (Faris \& Felmlee, 2011). En el caso del narcisismo, los resultados mostraron semejanza con estudios previos (Reijnties et al., 2016). Estas diferencias de sexo pueden interpretarse si las relacionamos con la posible intencionalidad de la agresión en relación al estatus social. Si bien es cierto que los chicos tienen una mayor tendencia a realizar comportamientos agresivos en busca de un dominio social entre sus compañeros y compañeras de clase (Fanti \& Kimonis, 2015), quizás ellas buscan ese dominio social a través de formas indirectas y sutiles de un protagonismo narcisista, que les conducen a alcanzar reconocimiento y éxito y, por tanto, mayor estatus social.

Respecto de la segunda hipótesis, se ha observado que existe relación entre la agresión y la ciberagresión y percibirse como popular y mostrar narcisismo. En este sentido, si bien en algunos estudios se observa que los agresores se caracterizan por su bajo apoyo social (Katzer et al., 2009), es ampliamente reconocido en la literatura científica que existe un perfil de agresor hábil socialmente, popular y aceptado, particularmente cuando la agresión es de tipo unidireccional, tal y como se da en el acoso escolar (Berger \& Caravita, 2016). De hecho, se ha asociado la implicación en agresión con la consecución de popularidad en la adolescencia. En este trabajo, nos encontramos con un perfil de implicados en agresión que, movidos por la obtención de popularidad dentro del grupo de iguales, se perciben como populares entre los chicos y narcisistas en el caso de las chicas. En el modelo de ciberagresión, las metas de popularidad no influyeron, lo que puede ser debido, en parte, al formato de preguntas incluidas en esta escala, más ligadas a comportamientos y situaciones que realizamos cara a cara y no de forma virtual.

La inclusión del narcisismo en los modelos de agresión y ciberagresión coincide con los hallazgos de los resultados de investigaciones 
anteriores con agresión cara a cara (Kiærvik \& Bushman, 2021), en los que se subraya que las conductas narcisistas ayudan a comprender, en cierta medida, por qué los chicos y chicas se implican en este tipo de fenómenos agresivos. No obstante, este trabajo supone una importante aportación en el estudio de las variables predictoras del ciberacoso, donde el narcisismo ha sido escasamente explorado.

En el caso de la tercera hipótesis, este estudio subrayó la influencia mutua entre la ciberagresión y la agresión. Si bien es destacable que los mayores niveles de relación se observan cuando la variable dependiente es la agresión en acoso escolar. Este resultado concuerda con los estudios sobre el solapamiento entre ambos tipos de violencia, cara a cara y virtual, donde se ha señalado que los adolescentes implicados en bullying tienen un mayor riesgo de estar implicados en ciberacoso que a la inversa (Chudal et al., 2021).

Estos resultados refuerzan la tesis mantenida referida a considerar que agresores y ciberagresores no acosan ni al azar ni como respuesta a otros conflictos, sino que básicamente su conducta parece dirigida a fortalecer su estatus social, su relevancia en el grupo (Navarro et al., 2015), sea en forma de control y dominio, sea en forma narcisística, tratando de ser protagonistas de admiración $y$, en definitiva, reconocimiento.

Los resultados obtenidos en este estudio nos permiten apoyar ciertas conclusiones relevantes a los efectos de la finalidad que este trabajo se propuso. Así, se ha cubierto el objetivo de describir los niveles de búsqueda de la popularidad, rasgos de narcisismo y agresión y ciberagresión de una buena muestra de adolescentes de Andalucía. Los resultados muestran elevados niveles de agresión y ciberagresión en aquellos chicos que puntúan alto en búsqueda de popularidad y en chicas que puntúan alto en narcisismo. Igualmente, este estudio aporta nuevas evidencias en el estudio del acoso y ciberacoso, donde el narcisismo y la popularidad percibida son variables que requieren una especial atención, no solo para comprender la implicación en agresión, sino también la ciberagresión.
Estos resultados nos invitan a concluir que si lo que se busca, en alguna medida, mediante la conducta agresiva injustificada, es lograr mayor estatus social, ser o percibirse como popular (ellos), ser un poco especial - percibirse como interesante (ellas), habría que prestar más atención a la dinámica interpersonal de los iguales en general, su microcultura, sus normas y convenciones, sus motivaciones e intereses, que quizás subyacen a comportamientos agresivos con sus iguales que deberían ser evitados. Se trata de conseguir, educativamente, eliminar que el acoso escolar refuerce la búsqueda de popularidad, relevancia y estatus social. Ello podría incidir en cambiar la actitud de los agresores (Garmendia et al., 2019). No cabe duda de que, reconocido el problema, la educación debe disponer de alternativas para alcanzar la popularidad y relevancia social buscada mediante la competencia atlética, artística, intelectual, etc., por ejemplo. Para ello se hace necesario además trabajar con la familia y la comunidad para revisar y cuestionar los valores que definen nuestra sociedad, muchos de los cuales van dirigidos a la búsqueda de poder y éxito y que pueden estar en la base de la explicación de los comportamientos dominantes, inmorales e injustificados.

Este estudio cuenta con algunas limitaciones. En primer lugar, los resultados presentados pertenecen a una muestra que, a pesar de ser amplia, forma parte de una región geográfica concreta. Sería de interés replicar este estudio en otras regiones y países que permitiera explorar las posibles similitudes y diferencias culturales. En segundo lugar, en este trabajo se ha explorado la popularidad percibida a través de dos ítems. Sería de interés considerar otros indicadores de medición de la popularidad, más allá del auto-informe, que incluya la percepción de los iguales, docentes y familias. Ello permitiría disponer de un constructo más amplio y compresivo del concepto popularidad. Igualmente, es necesario avanzar hacia la medición del constructo narcisismo con instrumentos que recojan situaciones que se aproximen tanto al contexto de la vida social directa como virtual 
de los escolares, así como tener en cuenta la multidimensionalidad del constructo (Fan et al., 2019). Pero aun con las limitaciones enunciadas, el trabajo tiene la potencialidad de abrir un campo de investigación que se dirige a buscar nuevos modelos explicativos de la compleja dinámica interpersonal y comunicativa de los iguales que se refleja en fenómenos como el acoso escolar y ciberacoso.

\section{- Conflicto de intereses}

Los autores declaran no tener ningún conflicto de interés.

\section{REFERENCIAS}

American Psychiatric Association. (2013). Diagnostic and statistical manual of mental disorders (DSM-5®). American Psychiatric Pub.

Berger, C., \& Caravita, S. C. S. (2016). Why do early adolescents bully? Examining latent profiles of behavior, social status, Machiavellianism, and empathy. Journal of youth and adolescence, 44(12), 22302244.

Card, N. A., Stucky, B. D., Sawalani, G. M., \& Little, T. D. (2008). Direct and indirect aggression during childhood and adolescence: A meta-analytic review of gender differences, intercorrelations, and relations to maladjustment. Child Development, 79, 1185-1229. https://doi.org/10.1111/i.1467. 8624.2008.01184.x

Cerezo, F., Sánchez, C., Ruiz, C., \& Arense, J. J. (2015). Roles en bullying de adolescentes y preadolescentes y su relación con el clima social y los estilos educativos parentales. Revista de Psicodidáctica, 20, 139-155. https://doi. org/10.1387/RevPsicodidact. 11097

Chudal, R., Tiiri, E., Klomek, A. B., Ong, S. H., Fossum, S., Kaneko, H., ... \& Sourander, A. (2021). Victimization by traditional bullying and cyberbullying and the combination of these among adolescents in 13 European and Asian countries. European Child \& Adolescent Psychiatry, 1-14. https://doi.org/10.1007/s00787. $\underline{021-01779-6}$

Cillessen, A. H. N., \& Mayeux, L. (2004).
From censure to reinforcement: Developmental changes in the association between aggression and social status. Child Development, 75, 147-163. https://doi.org/10.1111/i.14678624.2004.00660.x

Cillessen, A. H., Mayeux, L., Ha, T., de Bruyn, E. H., \& LaFontana, K. M. (2014). Aggressive effects of prioritizing popularity in early adolescence. Aggressive Behavior, 40(3), 204-213. https://doi. $\mathrm{org} / 10.1002 / \mathrm{ab} .21518$

Del Rey, R., Casas, J., Ortega-Ruiz, R., Schultze-Krumbholz, A., Scheithaver, H., Smith, P., Thompson, F., Barkoukis, V., Tsorbatzoudis, H., Brighi, A., Guarini, A., Pyalski, J., \& Plichta, P. (2015). Structural validation and cross-cultural robustness of theEuropean Cyberbullying Intervention Project Questionnaire. Computers in Human Behavior, 50, 141-147. https://doi.org/10.1016/i. chb.2015.03.065

Dumas, T. M., Davis, J. P., \& Ellis, W. E. (2019). Is it good to be bad? A longitudinal analysis of adolescent popularity motivations as a predictor of engagement in relational aggression and risk behaviors. Youth and Society, 51(5), 659-679. https://doi. org/10.1177/0044118X17700319

Fan, C. Y., Chu, X. W., Zhang, M., \& Zhou, Z. K. (2019). Are narcissists more likely to be involved in cyberbullying? Examining the mediating role of selfesteem. Journal of Interpersonal Violence, 34(15), 3127-3150. https://doi. org/10.1177/0886260516666531

Fanti, K. A., \& Henrich, C. C. (2015). Effects of self-esteem and narcissism on bullying and victimization during early adolescence. The Journal of Early Adolescence, 35(1), 5-29. https://doi. org/10.1177/0272431613519498

Faris, R., \& Felmlee, D. (2011). Status struggles: network centrality and gender segregation in same- and cross-gender aggression. American Sociological Review, 76, 48-73. https://doi. org/10.1177/0003122410396196

Fernández-Montalvo, J., Peñalva, A. \& Irazabal, I. (2015). Hábitos de uso y conductas de riesgo en Internet en la 
preadolescencia. Comunicar, 22(44). https://doi.org/10.3916/C44-2015-12

Garaigordobil, M. (2011). Prevalence and consequences of cyberbullying: A review. International Journal of Psychology and Psychological Therapy, 17 (2), 233-254.

Garmendia, M., Jiménez, E., \& Larrañaga, N. (2019). Bullying and cyberbullying: Victimisation, harassment, and harm. The need to intervene in the educational centre. Revista Española de Pedagogía, 77(273), 295-312. https://doi.org/10.22550/ REP77-2-2019-08

Golmaryami, F. N., \& Barry, C. T. (2010). The associations of self-reported and peer-reported relational aggression with narcissism and self-esteem among adolescents in a residential setting. Journal of Clinical Child and Adolescent Psychology, 39, 128-133. https://doi. org/10.1080/15374410903401203

Hendin, H. M., \& Cheek, J. M. (1997). Assessing hypersensitive narcissism: A reexamination of Murray's narcissism scale. Journal of Research in Personality, 31, 588-599.

Holt, M. K., \& Espelage, D. L. (2012). A review of research on bullying and peer victimization in school: An ecological system analysis. Aggression and violent behavior, 17(4), 311-322. https://doi. org/10.1016/i.avb.2012.03.003

Hymel, S., McClure, R., Miller, M., Shumka, E., \& Trach, J. (2015). Addressing school bullying: Insights from theories of group processes. Journal of Applied Developmental Psychology, 37, 16-24. https://doi.org/10.1016/i. appdev.2014.11.008

Katzer, C., Fetchenhaver, D., \& Belschak, F. (2009). Cyberbullying: who are the victims? A comparison of victimization in internet chatrooms and victimization in school. Journal of Media Psychology, 21 (1), 25-36. https://doi.org/10.1027/18641105.21 .1 .25

Kiærvik, S. L., \& Bushman, B. J. (2021). The link between narcissism and aggression: A meta-analytic review. Psychological Bulletin. Advance online publication. https://doi.org/10.1037/bul0000323

Kleiser, M., \& Mayeux, L. (2021).
Popularity and Gender Prototypicality: An Experimental Approach. Journal of Youth and Adolescence, 50(1), 144-158. https://doi.org/10.1007/s10964-020-

01344-5

Krizan, Z., \& Herlache, A. D. (2018).

The narcissism spectrum model: $A$ synthetic view of narcissistic personality. Personality and Social Psychology Review, 22(1), 3-31. https://doi. org/10.1177/1088868316685018

Laninga-Wijnen, L., Harakeh, Z., Garandeau, C. F., Dijkstra, J. K., Veenstra, R., \& Vollebergh, W. A. (2019). Classroom popularity hierarchy predicts prosocial and aggressive popularity norms across the school year. Child development, 90(5), e637-e653. https://doi.org/10.1111/ cdev. 13228

Malamut, S. T., van den Berg, Y. H. M., Lansu, T. A. M., \& Cillessen, A. H. N. (2020). Bidirectional associations between popularity, popularity goal, and aggression, alcohol use and prosocial behaviors in adolescence: A 3-year prospective longitudinal study. Journal of Youth and Adolescence, 50, 298-313. https://doi.org/10.1007/s10964-02001308-9

Miller, J. D., Campbell, W. K., \& Pilkonis, P. A. (2007). Narcissistic personality disorder: Relations with distress and functional impairment. Comprehensive Psychiatry, 48(2), 170-177. https://doi. org/10.1016/i.comppsych.2006.10.003 Montero, I., \& León, O. G. (2007). A guide for naming research studies in Psychology. International Journal of Clinical and Health Psychology, 7, 847-862.

Morf, C. C., \& Rhodewalt, F. (2001). Unraveling the paradoxes of narcissism: a dynamic self-regulatory processing model. Psychological Inquiry, 12, 177 196.

Navarro, R., Yubero, S., \& Larrañaga, E. (2015). Psychosocial Risk Factors for Involvement in Bullying Behaviors: Empirical Comparison between Cyberbullying and Social Bullying Victims and Bullies. School Mental Health, 7, 235-248. https://doi.org/10.1007/ s12310-015-9157-9 
Olweus, D. (1999). Sweden. The nature of school bullying: A cross-national perspective. London \& New York: Routledge.

Ortega-Ruiz, R., Del Rey, R., \& Casas, J. A. (2016). Evaluar el bullying y el cyberbullying validación española del EBIP-Q y del ECIP-Q. Psicología Educativa, 22, 71-79. https://doi. org/10.1016/i.pse.2016.01.004

Ortega-Ruiz, R., \& Zych, I. (2016). La ciberconducta y la psicología educativa: retos y riesgos. Psicología Educativa, 22(1), 1-4. https://doi.org/10.1016/i. pse.2016.04.001

Pauletti, R. E., Menon, M., Menon, M., Tobin, D. D., \& Perry, D. G. (2012). Narcissism and adjustment in preadolescence. Child Development, 83, 831-837. https://doi.org/10.1111/i.14678624.2011.01731.x

Poorthuis, A. M., Slagt, M., van Aken, M. A., Denissen, J. J., \& Thomaes, S. (2021). Narcissism and popularity among peers: A cross-transition longitudinal study. Self and identity, 20(2), 282-296. https://doi. $\mathrm{org} / 10.1080 / 15298868.2019 .160957$ $\underline{5}$

Pouwels, J. L., van Noorden, T. H., Lansu, T. A., \& Cillessen, A. H. (2018). The participant roles of bullying in different grades: Prevalence and social status profiles. Social Development, 27(4), 732-747. https://doi.org/10.1111/ sode. 12294

Reijnties, A., Vermande, M., Goossens, F. A., Olthof, T., Van de Schoot, R., Aleva, L., \& Van der Meulen, M. (2016). Narcissism, Bullying and Social Dominance in Youth: A Longitudinal Analysis. Journal of Abnormal Child Psychology, 44, 63-74. https://doi.org/10.1007/s10802-0159974- 1

Ripoll, C., Salazar, J., \& Bobes, J. (2010). Validez de la versión española de la Hypersensitive Narcissism Scale (HSNS) en una Unidad de Conductas Adictivas. Adicciones, 22(1), 29-36.
Romera, E. M., Casas, J. A., GómezOrtiz, O., \& Ortega-Ruiz, R. (2019). Moral domain as a risk and protective factor against bullying. An integrating perspective review on the complexity of morality. Aggression and Violent Behavior, 45, 75-82. https://doi.org/10.1016/i. avb.2018.07.005

Ryan, A. M., \& Shim, S. S. (2008). An exploration of young adolescents' social achievement goals and social adjustment in middle school. Journal of Educational Psychology, 100, 672-687. https://doi. org/10.1037/0022-0663.100.3.672

Smahel, D., Machackova, H., Mascheroni, G., Dedkova, L., Staksrud, E., Ólafsson, K., Livingstone, S., \& Hasebrink, U. (2020). EU Kids Online 2020: Survey results from 19 countries. EU Kids Online 2020.

Smith, P. K., Mahdavi, J., Carvalho, M., Fisher, S., Russell, S., \& Tippet, N. (2008). Cyberbullying: Its nature and impact in secondary school pupils. Journal of Child Psychology and Psychiatry, 49, 376385. https://doi.org/10.1111/i.14697610.2007.01846.x

Thomaes, S., \& Brummelman, E. (2015). Narcissism. In D. Cicchetti (Ed.), Developmental Psychopathology. New York: Wiley.

Washburn, J. J., McMahon, S. D., King, C. A., Reinecke, M. A., \& Silver, C. (2004). Narcissistic features in young adolescents: Relations to aggression and internalizing symptoms. Journal of Youth and Adolescence, 33, 247$260 . \quad$ https://doi.org/10.1023/ B: JOYO.0000025323.94929.d9

Zych, I., Ortega-Ruiz, R., \& Del Rey, R. (2015). Scientific research on bullying and cyberbullying: Where have we been and where are we going. Agression and Violent Behavior, 24, 188-198. https:// doi.org/10.1016/i.avb.2015.05.015 